\title{
COMPARATIVO DE RENDIMIENTO DE OCHO CLONES DE YUCA (Manihot esculenta, Crantz)
}

\author{
N. Pezo P. \\ W. Vásquez R.*
}

\section{RESUMEN}

Con el objetivo de comparar el rendimiento de raíces reservantes, se evaluaron ocho clones de yuca (Manihot esculenta, Crantz), bajo las condiciones de suelo y clima de Iquitos.

El experimento se llevó a cabo en un Diseño de Bloques al Azar, en el Campo Experimental Agrícola de la Facultad de Agronomía-UNAP, ubicado en Zúngaro Cocha a $25 \mathrm{~km}$. al S.O. de la ciudad de Iquitos.

De acuerdo a los resultados obtenidos se concluye que el clon "A marilla" fue el más rendidor, seguido de los clones $Z_{2}-82$ y "Zevallo negro", demostrando alta capacidad de producción.

\section{INTRODUCCION}

La yuca (Manihot esculenta, Crantz), ocupa un lugar importante en la agricultura de las regiones de clima tropical, no solo por su empleo en la alimentación humana y animal, sino también por su uso en la industria. Es la más eficiente en la producción de alimento energético produciendo por área más calorías que el maíz, arroz y tubérculos, posee bajo contenido de proteínas (menos del $20 \%$ ), en peso fresco y $15 \%$ en los cogollos y hojas tiernas.

Varios factores contribuyen a aumentar el interés en el cultivo de la yuca: su gran distribución, su amplia aceptación y adaptabilidad, con un gran potencial genético para su mejoramiento en rendimiento y calidad.

Teniendo en cuenta estas apreciaciones, se realizó el presente trabajo, cuyo principal objetivo fue probar comparativamente ocho

* Instituto de Investigaciones de la Amazonía Peruana-IIAP. Av. Abelardo Quiñones Km. 2.5 Apdo. 784. Iquitos-Perú.

** Ex-Docente de la Facultad de Agronomía. Universidad Nacional de la Amazonía Peruana. Samanez Ocampo 185, Aptdo. 496. Iquitos-Perú 
clones de yuca, que proporcionen mejores rendimientos por unidad de superficie y tratar de esta forma de suplir en parte las deficiencias alimenticias del poblador amazónico.

\section{REVISION DE LITERATURA}

Montaldo (1979), indica que los rendimientos de yuca son muy diversos y dependen de la naturaleza de las variedades, la duración del período vegetativo, las condiciones del medio ambiente y la forma del cultivo.

Brambilla (1972), establece según referencia de agricultores de la Costa Central del Perú, que con la variedad "Amarilla", se obtienen 15 t/ha; mientras que en Iquitos con la variedad "Blanca", se obtiene un rendimiento de $25 \mathrm{t} / \mathrm{ha}$, de igual forma se logra con la "Criolla" en Pucallpa.

Delgado y Rosas (1977), manifiestan que en la Estación Experimental de "El Porvenir", Tarapoto, los cultivos que destacaron en rendimiento fueron "Auquina amarilla", con 25 t/ha y "Rumo maqui", con 17 t/ha.

Ríos (1981), en la zona de Iquitos, en un experimento con fertilización y utilizando la variedad "Motelo rumo", obtuvo un rendimiento de raíces de 47,246 kg/ha.

Rodríguez (1984), trabajando con diez variedades de yuca en condiciones de suelo y clima de lquitos, encontró que el mayor rendimiento obtenido fue de $42,500 \mathrm{~kg} / \mathrm{ha}$ para la variedad "Palo negro" y el menor fue de $8,150 \mathrm{~kg} / \mathrm{ha}$ para la variedad "Ungurahui".

\section{MATERIALES Y METODOS}

El experimento, se llevó a cabo en el Campo Experimental Agrícola de la Facultad de Agronomía-UNAP, localizado en Zúngaro Cocha, aproximadamente a $25 \mathrm{~km}$. al S.O. de la ciudad de lquitos.

Durante la conducción del experimento, el cultivo recibió una precipitación que varió de 62.7 a $487 \mathrm{~mm}$; mientras que la temperatura

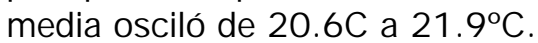

El suelo experimental fue de textura franco arcillo arenoso, el pH muy ácido y un contenido medio de materia orgánica. 
Los tratamientos en estudio fueron 8 clones de yuca proporcionados por el Programa de Yuca del CIPA XVI, Iquitos, los mismos que fueron instalados en Diseño de Bloques al Azar con tres repeticiones. La parcela experimental tuvo una longitud de $6.0 \mathrm{~m}$. y un ancho de $4.0 \mathrm{~m}$.; siendo el área útil cosechable de $10.0 \mathrm{~m}^{2}$. Para la siembra se empleó estacas de $15 \mathrm{~cm}$. de longitud, sembrándose en posición oblicua y con distanciamiento de $1.0 \times 1.0 \mathrm{~m}$. siembra.

La cosecha se efectuó a los 10 meses después de realizada la

La observación registrada y sometida al cálculo estadístico fue el rendimiento de raíces tuberosas.

\section{RESULTADOS Y DISCUSION}

\section{RENDIMIENTO DE RAICES TUBEROSAS}

El rendimiento de las raíces, no alcanzaron diferencias significativas según el Análisis de Variancia, que se muestra en el Cuadro 1.

\section{Cuadro 1}

Análisis de variancia para rendimiento de raíces en kilogramos por parcela de 8 clones de yuca.

\begin{tabular}{|l|r|rl|}
\hline F. de V. & G L & C M & Fc \\
& & & \\
\hline Bloques & 2 & 61.345 & 0.806 \\
Tratamientos & 7 & 48.971 & 0.643 \\
Error & 14 & 76.098 & 0.643 \\
\hline
\end{tabular}

Con respecto al rendimiento de las raíces tuberosas, creemos que es el carácter más importante y constituye una característica clonal, aunque influenciado por los factores ambientales y culturales; en nuestra zona por ejemplo, los agricultores siembran cuando menos más de un clon de yuca, sin ningún cuidado o técnica, en tales condiciones los rendimientos son bajos. 
En nuestro estudio, este carácter no ha mostrado estadísticamente variaciones significativas, tal como se muestra en el Cuadro 2, pero se observa una fuerte diferencia de producción.

\section{Cuadro 2}

Rendimiento promedio de raíces en kilogramos por hectárea de 8 clones de yuca. Resultados de la Prueba de Duncan(*)

\begin{tabular}{|c|l|l|}
\hline U M & \multicolumn{1}{|c|}{ Tratamientos } & Rendimientos \\
\hline 1 & Amarilla & $26760 \mathrm{a}$ \\
2 & $Z_{2}-82$ & $23760 \mathrm{a}$ \\
3 & Zevallo negro & $21030 \mathrm{a}$ \\
4 & Zi $-81-\mathrm{R}$ & $20710 \mathrm{a}$ \\
5 & Lobera & $20580 \mathrm{a}$ \\
6 & Umisha rumo blanco & $17300 \mathrm{a}$ \\
7 & Z1 $-81-\mathrm{B}$ & $16660 \mathrm{a}$ \\
8 & Motelo rumo & $13830 \mathrm{a}$ \\
\hline C.V. = 43.6\% & \multicolumn{2}{|c|}{ Promedio $=20003.75$} \\
\hline
\end{tabular}

Promedios que tienen las mismas letras no muestran diferencias significativas.

El clon "Amarilla" produjo más que el rendimiento del clon "Motelo rumo" que ocupa el último lugar; los clones $Z_{2}-82$, "Zevallo negro", $Z_{1}$ 81 - R y "Lobera" con rendimientos que superan el promedio general; y los demás, "Umisha rumo blanco" y $Z_{1}-81$ - B, con rendimiento infereriores al promedio general.

Esta variación en el rendimiento encontrado no puede atribuirse de modo directo a los factores ambientales ni a las prácticas culturales, puesto que en este último caso todos los clones han recibido el mismo tratamiento, sino debe presumirse a una capacidad de rendimiento de cada clon. Estos resultados demuestran a la vez la existencia de una variación clonal en el rendimiento de las yucas estudiadas, demostrando así mismo que es posible seleccionar clones de alta capacidad de rendimiento que permitiría aumentar la producción de yuca por unidad de superficie. 


\section{CONCLUSIONES Y RECOMENDACIONES}

No obstante que las pruebas estadísticas indican ausencia de significancia, se demuestra la existencia de una gran variabilidad, lo que permite seleccionar clones de yuca de alta producción.

El clon "Amarilla", resultó ser la de mayor rendimiento de raíces tuberosas. Merece destacarse el comportamiento de los clones $Z_{2}-82$ y "Zevallo negro" con rendimientos superiores al promedio general obtenido por los clones estudiados.

Se recomienda repetir el experimento, incluyendo otros clones que se cultivan en la zona, a fin de conocer la capacidad de producción y seleccionar las más sobresalientes.

\section{BIBLIOGRAFIA}

BRAMBILLA, A. J . 1972. Cultivos alimenticios y cultivo de la yuca. La Molina: $47 \mathrm{p}$.

CALZADA BENZA J. 1970. Métodos Estadísticos para la Investigación. 3a. ed. Lima: Editorial J urídica. $645 \mathrm{p}$.

DELGADO E. y ROSAS C. 1977. Resultados de la Investigación recomen daciones para su cultivo en el país. Yuca. Informe especial № 65. Lima (Perú): 25 p.

MONTALDO, A. 1979. La yuca o mandioca. San J osé (Costa Rica): Ed. IICA, 386 p.

RIOS, S. O. 1981. Fertilización orgánica con suplemento mineral en el cultivo de la yuca (Manihot esculenta, Crantz), en lquitos. Tesis Ingo Agr. Universidad Nacional de la Amazonía Peruana, Programa Académico de Agronomía, 69 p.

RODRIGUEZ, N. 1984. Estudio de nemátodos radiculares de 10 variedades de yuca (Manihot esculenta, Crantz), en Iquitos.Tesis Ingo Agr. Universidad Nacional de la Amazonía Peruana, Programa Académico de Agronomía. 